\title{
Significant overexpression of Hsp110 gene during colorectal cancer progression
}

\author{
O. SLABY ${ }^{1,3}$, K. SOBKOVA ${ }^{1,3}$, M. SVOBODA ${ }^{2,3}$, I. GARAJOVA ${ }^{2,3}$, P. FABIAN ${ }^{1}$, R. HRSTKA ${ }^{1}$, R. NENUTIL ${ }^{1}$, \\ M. SACHLOVA ${ }^{2,3}$, I. KOCAKOVA ${ }^{2,3}$, J. MICHALEK ${ }^{3,4}$, T. SMERDOVA ${ }^{1}$, D. KNOFLICKOVA ${ }^{1}$ and R. VYZULA ${ }^{2,3}$ \\ Departments of ${ }^{1}$ Oncological and Experimental Pathology, ${ }^{2}$ Comprehensive Cancer Care, Masaryk Memorial Cancer \\ Institute, ${ }^{3}$ Medical Faculty, Masaryk University, ${ }^{4}$ University Cell Immunotherapy Center, Brno, Czech Republic
}

Received July 31, 2008; Accepted September 18, 2008

DOI: $10.3892 /$ or_00000346

\begin{abstract}
Colorectal cancer (CRC) is one of the most frequent malignant diseases in the world. Metastatic spread of the cancer to the lymph nodes is a crucial factor for progression and therapeutic management of the disease. We analysed gene expression profiles of CRC patiens by lowdensity cancer-focused oligonucleotide microarrays to identify new predictive markers of the extent of the disease and for better understanding of CRC progression. Relative expression levels of 440 genes known to be involved in cancer biology were obtained by low-density oligonucleotide microarrays from 20 tumor samples. Statistical analysis of gene expression data identified 3 genes (HSP110, HYOU1 and TCTP) significantly up-regulated in primary tumors of patients who developed lymph node metastasis. We have shown, for the first time, that up-regulation HSP110 and HYOU1 expression is associated with lymph node involvement in CRC. We validated the differences in HSP110 expression in an independent group of 30 patients of all clinical stages by real-time PCR. We identified significant up-regulation of HSP110 expression in colorectal tumors compared to adjacent non-tumoral tissue $(\mathrm{p}<0.0003)$. We observed significant differences of HSP110 gene expression between metastatic and localized disease $(p=0.031)$ and negative trend of HSP110 gene expression and overall survival of CRC patients. We suggest that HSP110 gene is a promising molecular predictor in CRC.
\end{abstract}

\section{Introduction}

Colorectal cancer (CRC) is one of the most frequent malignant diseases in the world. With the incidence rate $\sim 78$ per 100,000 people, the Czech Republic is a country with

Correspondence to: Dr Ondrej Slaby, Masaryk Memorial Cancer Institute, Department of Oncological and Experimental Pathology, Zluty kopec 7, 65653 Brno, Czech Republic

E-mail: slaby@mou.cz

Key words: colorectal cancer, staging, lymph nodes, progression, gene expression profiles, Hsp110 one of the highest incidence of CRC in the world and the highest in Europe (1). The prognosis of these patients depends largely on the extent of the disease and a possibility of curative surgical intervention which is feasible only in patients with disease limited to the primary tumor and regional lymph nodes. Spread of the cancer to lymph nodes has been considered a crucial factor for progression and further therapeutic management of the disease. While in the case of clinical stage I and IV, the prognostic significance of the TNM classification is evident, for the group of patients diagnosed at stages II (without dissemination to regional lymph nodes) and III (with dissemination to lymph nodes) the prognostic information of clinical stage is much lower. The fact, that $30 \%$ of patients with clinical stage II of CRC will progress within five years and only $45 \%$ of the patients with clinical stage III will reach a 5-year survival, although undergoing radical surgery, implies that recent staging of CRC based on TNM classification is not optimal and failed for a significant proportion of non-advanced CRC patients. This situation is caused by two factors: firstly, understaging of regional lymph node status for the reason of insufficient number of resected and/or examined lymph nodes, secondly, the TNM classification does not include biological characteristics and predictors of tumor behaviour. Multicentric studies based on cancer registers, in which thousands of patients are included, have proved that number of examined lymph nodes has a significant impact on the prognosis, even if lymph nodes were not infiltrated by tumor cells (2-7). For the purposes of the TNM classification UICC (International Union Against Cancer) has recommended that the histological examination of samples from regional lymphadenectomy should involve twelve or more regional lymph nodes. It is acceptable, in case of a low number of examined lymph nodes, to use pN0 for lymph node status classification. From this point of view, results of meta-analysis focused on the number of examined lymph nodes are very interesting. Approximately $20-30 \%$ of clinical samples are not examined adequately according to the UICC data (2). Clinical consequences of the $\mathrm{pN}$ understaging are more important in respect to growing efficiency of colorectal cancer treatment, which is mentioned usually in connection with biological therapy expected soon also in the adjuvant regimens. 
DNA microarray technology is currently the most effective and widespread technique used for gene expression studies. In recent years the number of studies related to this technology exponentially increases and utilization of DNA microarrays has markedly drifted from basic to clinical research. However, comparability and reproducibility of studies based on high-density DNA microarrays are notably affected by their technological diversity, and recent findings are not conclusive $(8,9)$.

This study aimed to find individual up-/down-regulated genes associated with progression and metastatic spread of colorectal cancers to the regional lymph nodes using lowdensity oligonucleotide microarrays spotted with genes known to be involved in cancer biology. We suppose that focusing on particular biological pathways may be more useful than genome-wide screening for our purposes and it is not associated with the need of large sample sizes as it is necessary for high-density microarrays (10). Molecular characterization of patients with high risk of cancer progression using this more economical and productive expression profiling method may improve our knowledge on cancer progression and dissemination and may also assist oncologists in treatment decision by selecting those patients who will need adjuvant chemotherapy and in the case of patients with rectal cancer may also amend indication for neoadjuvant treatment.

\section{Patients and methods}

Study population and tissue samples preparation. After informed consent was obtained, 50 patients (20 in the training group, 30 in the validation group) who underwent surgery at Masaryk Memorial Cancer Institute for the manifestation of CRC were included in this study. Patients indicated for preoperative radiation or/and chemotherapy or patients with hereditary CRCs (FAP and HNPCC) or microsatellite instability, according to imunohistochemistry of mismatch repair proteins MSH2, MLH1 and PMS2 (11), were not involved. Gender, patient age, tumor localization, histopathological grade and UICC (International Union Against Cancer) stage were used as conventional clinicopathological parameters and are listed in Table I. Under pathologist's supervision, 80 tissue samples were collected from surgically resected tissues, 50 samples from colorectal adenocarcinomas (total cell population was composed of at least $80 \%$ of tumor cells) and 30 from adjacent non-tumor mucosal tissues. Mesenteric and pericolous adipose tissue in colorectal specimens was investigated for lymph nodes and examined by serial sections for lymphatic metastases. Only patients with sufficient number of examined lymph nodes (>12) were included. Time from clamping of CRC arterial supply to resection was controlled and tissue fixation (RNAlater, Ambion, US) occurred within 15 min after surgical extirpation. All samples were stored in liquid nitrogen until RNA extraction.

RNA isolation. Samples were homogenized (Retch MM301) in sterile conditions before total RNA isolation. Total RNA isolation was performed with the RNeasy Isolation kit (Qiagen, US) according to the manufacturer's instructions.
Table I. Patient and tumor characteristics of 50 patients with colorectal cancer.

\begin{tabular}{lcc}
\hline Variable & Training group & Validation group \\
$\mathrm{N}$ & 20 & 30 \\
\hline Age & & \\
Mean & 70 & 63 \\
Range & $40-85$ & $45-84$ \\
Gender & & \\
Male & 12 & 16 \\
Female & 8 & 14
\end{tabular}

Tumor location

$\begin{array}{lrr}\text { Proximal } & 8 & 14\end{array}$

$\begin{array}{lll}\text { Distal } & 12 & 16\end{array}$

\section{Differentiation}

Well

Moderate

Poorly

$7 \quad 6$

9216

$4 \quad 8$

UICC classification

$\begin{array}{llc}\text { Stage I } & 0 & 5\end{array}$

Stage II $10 \quad 13$

Stage III $\quad 10 \quad 7$

Stage IV $\quad 0 \quad 5$

RNA concentration and purity were measured by UV spectrophotometry (A260:A280>2.0; A260:A230>1.8). RNA integrity was checked using Agilent 2100 Bioanalyzer and only non-degraded RNA characterized by RIN (RNA Integrity Number) $>7$ with no DNA contamination signs was processed (12).

Low-density oligonucleotide microarrays processing. Relative expressions of 440 genes known to be involved in cancer biology were obtained by use of low-density oligonucleotide microarrays (SuperArrays, Bethesda, MD, Cancer Array-EHS-802) according to manufacturer's recommendations. Total RNA (3 $\mu \mathrm{g})$ from respective samples was used as a template for one-round linear amplification and biotin-16UTP (Enzo Life Sciences, USA) labeling to produce biotinlabeled cRNA in sufficient amount using TrueLabelingAMP $^{\mathrm{TM}} 2.0$ (SuperArray, Bethesda). For purification of biotin-labeled cRNA ArrayGrade cRNA cleanup kit (SuperArray, Bethesda) was used. cRNA probes corresponding to the mRNA population were then denatured, and hybridization was carried out in GEAhyb hybridization solution (SuperArray, Bethesda) for $20 \mathrm{~h}$ to nylon membranes spotted with gene-specific 60-mer oligonucleotide fragments. Membranes were then washed in 2X SSC, $1 \%$ SDS twice for $15 \mathrm{~min}$ each, followed by $0.1 \mathrm{X}$ SSC, $0.5 \mathrm{X}$ SDS twice for 15 min each. Hybridized cRNA was detected by binding of biotin to avidin-phosphatase conjugate and 



Figure 1. Example of two low-density oligonucleotide arrays with different expression of Hsp110 (in red circle). (A) Expression profile of patient with disease disseminated to lymph nodes. (B) Expression profile of patient with localized disease.

chemiluminescence reaction with CDPstar (Tropix, Inc., Bedford, MA). For chemiluminescence image acquisition 16bit cooled CCD camera (Alpha Innotech, Ltd., USA) was used with exposure time $7 \mathrm{~min}$ and the highest resolution and no bining settings.

Image analysis, data normalization and statistics. Image analysis, background substraction and data normalization were carried out by web-based application GEArray Expression Analysis Suite (SuperArray, Bethesda). A grid was automatically set up for each image, and the alignment to the array of spots was checked. The spots were quantified by taking the mean of the intensities of their pixels. Background intensity for each spot was determined from the background surrounding the spot by taking the mean of the background area. Spot intensities were normalized to the intensity of housekeeping gene-GAPDH. For statistical analysis and visualization of normalized data TIGR MultiExperiment viewer 4.0 (The Institute for Genomic Research, USA) (13) and J-Express Pro 2.7 (MolMine AS, The Netherlands) were used. For identification of expression differences between two groups of patients Significance Analysis of Microarrays (SAM) method (14) was used with condition of at least two-fold difference of identified genes between two groups. The SAM method was used for a study with design 'two class unpaired data' and false discovery rate (FDR) was declared $\Delta=0.75$. Genes with the most significant changes identified by both methods were validated by realtime PCR on an independent group of patients.

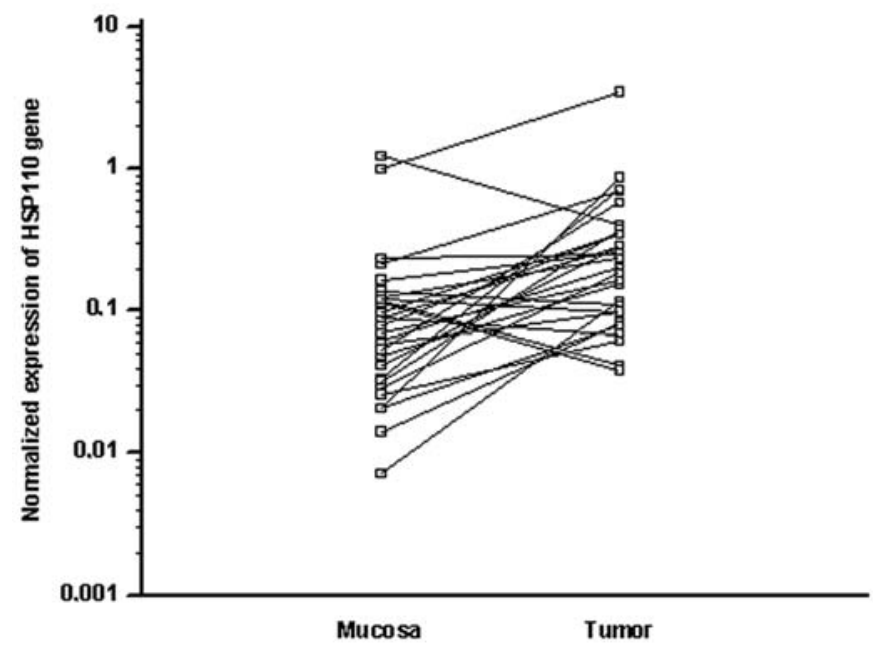

Figure 2. Gene expression of HSP110 in primary colorectal tumors and paired non-tumoral colorectal tissue (Wilcoxon paired test; $\mathrm{p}<0.0003$ ).

Reverse transcription and real-time PCR. cDNA was obtained by reverse transcription (Thermocycler Tgradient, Biometra, GE). Reaction mixture containing $1 \mu \mathrm{g}$ of total RNA, oligo d(T) primers (Generi Biotech, Czech Republic) and ribonuclease-free water was prepared. The mixture was incubated at $65^{\circ} \mathrm{C}$ for $5 \mathrm{~min}$, than cooled quickly on ice and 
Table II. Genes up-regulated in primary tumors of patients with metastatic spread to lymph nodes.

\begin{tabular}{llcccc}
\hline $\begin{array}{l}\text { Accession } \\
\text { Number }\end{array}$ & Symbol & Gene name & Previous report & $\begin{array}{c}\text { Ratio N+/N0 } \\
\text { microarrays }^{\text {a }}\end{array}$ & RatioN+/N0 PCR $^{\text {b }}$ \\
\hline NM_214530 & HSP110 & Heat shock protein 110 & 36,37 & 2.4 & 2.1 \\
NM_006389 & HYOU1 & Hypoxia up-regulated 1 & 31 & 2.2 & 1.05 \\
NM_112537 & TCTP & $\begin{array}{c}\text { Translationally controlled } \\
\text { tumor protein }\end{array}$ & 30 & 2.05 & 1.2 \\
\hline
\end{tabular}

$\mathrm{N}^{+}$, median expression of particular gene in patients with lymph nodes involvement - UICC stage III; N0, median expression of particular

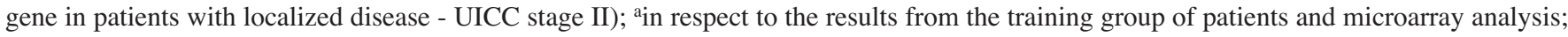
bin respect to the results from the validation group of patients and real-time PCR analysis.

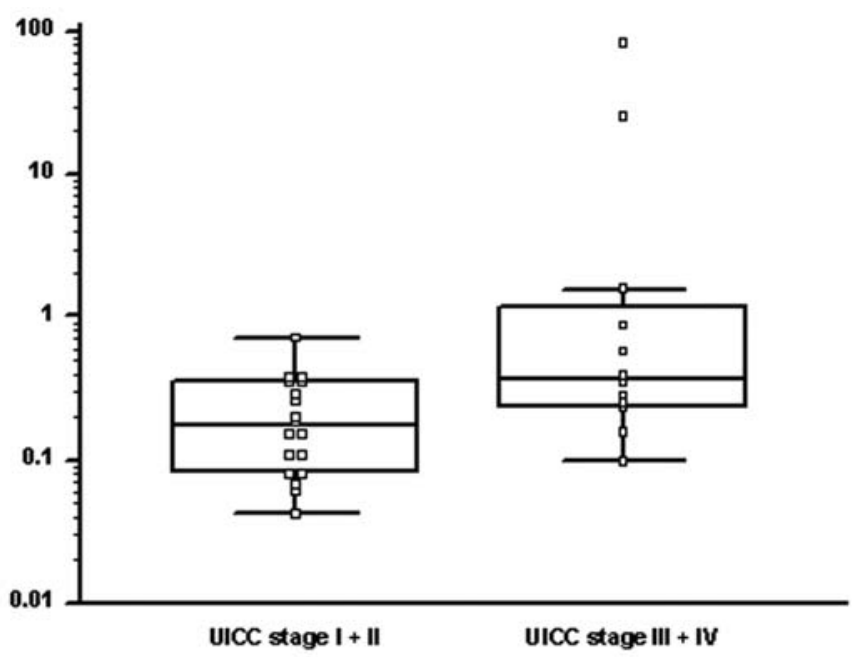

Figure 3. Gene expression of HSP110 in primary colorectal tumors of localized and metastatic disease in the validation group of patients (MannWhitney test; $\mathrm{p}=0.031$ ).

following components were added: 5X Reaction Buffer (Fermentas, USA), Ribonuclease Inhibitor (Fermentas), dNTP mix (Fermentas), Revert Aid ${ }^{\mathrm{TM}}$ M-MuLV H- Reverse Transcriptase (Fermentas) and ribonuclease-free water to $20 \mu \mathrm{l}$ final volume. The mixture was incubated for $120 \mathrm{~min}$ at $42^{\circ} \mathrm{C}$; the reaction was stoped by heating the mixture at $95^{\circ} \mathrm{C}$ for $5 \mathrm{~min}$ and chilled on ice.

The real-time PCR was performed using Applied Biosystems 7000 Sequence Detection System according to manufacturer's recommendations. PCR reaction mixture included $4 \mu \mathrm{l}$ RT product, 1X TaqMan Universal PCR Master mix and $1.25 \mu 1$ of primers and probe mix of the TaqMan expression assay (HSP110-Hs00971475_m1, HYOU1-Hs00197328_m1, TCTP-Hs02621289_g1; Applied Biosystems, USA). Reactions were incubated in 96-well optical plate at $95^{\circ} \mathrm{C}$ for $10 \mathrm{~min}$, followed by 40 cycles of $95^{\circ} \mathrm{C}$ for $15 \mathrm{sec}$ and $60^{\circ} \mathrm{C}$ for $10 \mathrm{~min}$. The $\mathrm{Ct}$ data were determined using default threshold settings. Samples were run in molecular biology of cancer.



Figure 4. Kaplan-Meier survival curves illustrating overall survival of CRC patients in the validation group on the basis of the HSP110 gene expression (long-rank p=0.1457).

Data analysis. Normalization is an essential step for the accurate quantification of mRNA levels and the process commonly used is the normalization of target gene according to the endogenous standard, such as housekeeping gene. Expression data in our study were normalized according to the expression of GAPDH (Catalog No. 402869; Applied Biosystems). Statistical differences between clinicopathologic parameters and mRNA levels were evaluated using non-parametric tests, Mann-Whitney U-test between two independent groups and Wilcoxon test between paired samples. The long-rank test was used to measure the difference in Kaplan-Meier curves. $\mathrm{P}<0.05$ were considered to be significant. All calculations were performed with the software Statistica version 6.0 (StatSoft Inc., USA).

\section{Results}

Identification of genes associated with lymph node metastasis. Low-density oligonucleotide microarrays were 
Table III. HSP110 gene expression in patients with localized (UICC stage I, II) and metastatic (UICC stage III, IV) colorectal cancer.

\begin{tabular}{lllllll}
\hline Gene & Disease & N & Median & Min & Max & Mann-Whitney test \\
\hline Hsp110 & Localized & 18 & 0.1741 & 0.0814 & 0.3536 & $\mathrm{p}=0.031$ \\
& Metastatic & 12 & 0.3487 & 0.1719 & 20.104 & \\
\hline
\end{tabular}

HSP110 gene expression levels are normalized.

used for the expression detection of 440 genes potentially involved in molecular biology of cancer. Relative gene expression levels of these 440 genes were obtained from 20 primary colon cancer samples of patients included in the training group of patients with no prior chemotherapy or radiotherapy. Examples of two oligonucleotide microarrays are shown in Fig. 1, indicating identified changes in expression of HSP110. Mean of the empty spot signals were used for background correction and intensity of GAPDH spot was applied for data normalization because its expression was the most stable from the panel of housekeeping genes localized on the microarray on the studied group of patients. Positive signal intensities ( $>10 \%$ of median value) were detected in $>40 \%$ of spots on the microarrays for twenty tumor samples and ranged from 41 to $75 \%$. Data analysis based on the SAM (Significance Analysis of Microarrays) method identified 3 genes (HSP110, HYOU1 and TCTP) with significant and at least 2-fold up-regulation in primary tumors of patients with metastatic spread to regional lymph nodes (see Table II). Interestingly, we did not observe significant changes in expression of several genes recently discovered to be associated with higher risk of lymph node metastasis such as VEGFA, p53, p21, MDM2 and KRAS.

Validation of the experiment and statistics. To examine the reliability of our microarray data, gene expressions of HSP110, HYOU1 and TCTP were further validated by more precise real-time PCR on the validating group of patients (see Table I). Comparison of the results between microarrays (training group of patients) and real-time PCR (validating group of patients) are shown in Table II. Only changes in the expression of HSP110 observed on the microarrays were verified by real-time PCR on the independent group of patients. HSP110 gene was chosen for further studies. In the validating group of patients also non-tumoral adjacent tissues were analyzed. Up-regulation of the HSP110 gene in tumors in comparison to adjacent non-tumoral mucosa was statistically significant $(\mathrm{p}<0.0003$; see Fig. 2$)$. For the fist time, we observed significant differences of HSP110 gene expression between metastatic and localized disease $(p=0.031$, see Table III) and negative trend of HSP110 gene expression levels and overall survival of colorectal cancer patients (see Fig. 4).

\section{Discussion}

The benefit of adjuvant chemotherapy for colorectal cancer (CRC) seems to be limited to patients with UICC clinical stage III, where the cancer has metastasized to lymph nodes at the time of diagnosis. On the other hand, usage of the TNM classification for determining the patients who need adjuvant chemotherapy is not optimal and failed for the significant proportion of not-advanced CRC patients, especially because of understaging of regional lymph node status, as well as TNM classification does not include biological characteristics and predictors of tumor behaviour (2-7).

Gene expression profiling with DNA microarrays has shown molecular changes that can determine carcinogenesis and cancer progression and ultimately the prognosis of a variety of tumors (15). In case of colorectal cancer, DNA microarrays were used in more than seventy studies during the last six years. These studies show efficiency of this technology to distinguish tumor from normal colonic tissue and classify tumors according to their anatomic localization $(16,17)$ and microsatellite status $(17,18)$. Subsequent reports demonstrate abilities of gene expression profiles to determine molecular signature of metastatic disease in primary tumors $(20,21)$ and predict therapy response and disease prognosis (22-25). A primary objective of this study was to identify differentially expressed genes in colorectal cancer, to search for specific gene-expression patterns associated with colorectal cancer progression, especially with metastatic spread to the regional lymph nodes. Scarce data exist focused on gene expression changes in CRC patients with lymph nodes metastases (26-29) with a little or null overlap between groups of identified genes in the independent studies with analogous design. The cause of this discouraging fact lies in the technological variability of DNA microarrays platforms, mainly in the algorithms used for designing probes by different manufacturers.

In the current study, we identified 3 genes (HSP110, HYOU1, TCTP) with significant, at least 2-fold upregulation, in the group of patients with cancer metastasizing to the lymph nodes in comparison to patients with localized disease by use of the low-density oligonucleotide microarrays. Up-regulation of HSP110 and HYOU1 gene expression in the CRC with lymph node involvement are shown for the first time. TCTP was found to be induced by mitogenic stimulation and it is thought to play an important role in cell growth. In the colon cancer cells high TCTP mRNA expression was related to the rapid cell growth and, therefore, a high potential of tumorigenesis (30). Heat shock protein 110 (HSP110) and hypoxia up-regulated 1 (HYOU1/Orp150) are highly conserved molecular chaperones with the function in mediating protein folding, 
assembly and transport under conditions of various cellular stressors such as hypoxia. Many malignant solid tumors as well as CRC contain significant fractions of hypoxic cells as a consequence of inadequate vascularization. The presence of hypoxic cells in solid tumors has long been considered a problem in cancer treatment. Hypoxic tumor cells have been shown to be more resistant to radiotherapy and many conventional chemotherapeutic agents than their normoxic counterparts. Moreover, HYOU1 is directly involved in the induction of vascular endothelial growth factor (VEGF) and consequently in the tumor neoangiogenesis $(31,32)$, which is very important especially in regard to introduction of targeted biological therapy to clinical routine.

In the validation phase of our study only changes in HSP110 gene expression identified by microarray analysis were proved on the independent group of patients by quantitative real-time PCR (see Table II). HSP110 is a part of the large multiprotein complex with the most common heat shock protein HSP70. HSP70 and HSP90 are important regulators of the cellular proliferation, effective antiapoptotic factors and are involved in the cancer biology (3234). Interestingly, we have not identified significant changes in expression of HSP70 or HSP90 between studied groups of patients by microarrays analysis. Molecular action of HSP110 role in cancer cells is not yet fully understood. However, Hosaka et al used sucessfully small interfering RNA (siRNA)-mediated supression of HSP110 protein expression for in vitro apoptosis induction in HCT116 colon cancer cells (35). This siRNA-induced apoptosis was mediated through caspases, but not through the p53 tumor suppressor pathway, even though the HSP110 protein was bound to wild-type p53 protein in HCT116 cells. These findings suggest that the constitutive overexpression of HSP110 in cancer cells is involved in malignant transformation by protecting tumor cells from apoptosis (35). HSP110 protein is overexpressed in various human tumors, including colon cancer cells but not colorectal adenomas, thus suggesting that overexpression of HSP110 is a late event in colorectal adenoma-carcinoma sequence (36). In this study, higher levels of HSP110 gene expression were associated with metastatic disease in comparison to those in tumors of patients with localized disease. Our data are in agreement with results of Hwang et al, who observed higher frequency of HSP110 protein positivity in colorectal adenocarcinomas of advanced clinical stages (37).We observed significant upregulation of HSP110 gene expression in tumors compared to paired adjacent non-tumoral tissue (see Fig. 2) and negative trend of HSP110 mRNA levels with overall survival (see Fig. 4) of colorectal cancer patients.

In conclusion, as a result of the gene expression profile analysis by use of SAM method, we indentified 3 genes (HSP110, HYOU1 and TCTP) with significant and at least 2-fold up-regulation in primary colorectal tumors of patients with metastasis to regional lymph nodes. Expression changes of HSP110 gene were succesfully validated by real-time PCR on the independent group of patients and for the first time associated with metastatic CRC disease and negatively correlated to the worse overall survival of colorectal cancer patients. According to our results, supported by data published by others, we suggest that HSP110 gene is a promising molecular predictor in CRC. Its association with common clinicopathological data may help in the diagnosis of the lymph node metastatic involvement, identifying more aggressive tumors and in predicting the clinical outcome, thus, might help clinical oncologists in designing or selecting more aggressive therapies for subgroups of patients with colorectal cancer.

\section{Acknowledgements}

This work was supported by grant IGA NR 9076 from the Czech Ministry of Health and Project MZ0MOU2005.

\section{References}

1. Dusek L, Muzik J, Kubásek M, Koptíková J, Zaloudík J and Vyzula R: Czech national web portal of epidemiology of malignant tumours (online). Masaryk University, 2005, (cit. 2008-5-27). http://www.svod.cz.

2. Baxter NN, Virnig DJ, Rothenberger DA, et al: Lymph node evaluation in colorectal cancer patients: a population-based study. J Natl Cancer Inst 97: 219-225, 2005.

3. Lee HY, Choi HJ, Park KJ, et al: Prognostic significance of metastatic lymph node ratio in node-positive colon carcinoma. Ann Surg Oncol 14: 1712-1717, 2007.

4. Murphy J, Pocard M, Jass JR, et al: Number and size of lymph nodes recovered from Dukes B rectal cancers: correlation with prognosis and histologic antitumor immune response. Dis Colon Rectum 50: 1526-1534, 2007.

5. Sarli L, Bader G, Iusco D, et al: Number of lymph nodes examined and prognosis of TNM stage II colorectal cancer. Eur J Cancer 41: 272-279, 2005

6. Cianchi F, Palomba A, Boddi V, et al: Lymph node recovery from colorectal tumor specimens: recommendation for a minimum number of lymph nodes to be examined. World J Surg 26: 384-389, 2002.

7. Morris EJ, Maughan NJ, Forman D, et al: Who to treat with adjuvant therapy in Dukes' B/stage II colorectal cancer? The need for high quality pathology. Gut 56: 1419-1425, 2007.

8. Shih W, Chetty R and Tsao MS: Expression profiling by microarrays in colorectal cancer (Review). Oncol Rep 13: 517-524, 2005

9. Draghici S, Khatri P, Eklund AC, et al: Reliability and reproducibility issues in DNA microarray measurements. Trends Genet 22: 101-109, 2006.

10. Tibshirani R: A simple method for assessing sample sizes in microarray experiments. BMC Bioinformatics 7: 106, 2006.

11. Plevova P, Krepelova A, Papezova M, et al: Immunohistochemical detection of the hMLH1 and hMSH2 proteins in hereditary non-polyposis colon cancer and sporadic colon cancer. Neoplasma 51: 275-284, 2004.

12. Schroeder A, Mueller O, Stocker S, et al: The RIN: an RNA integrity number for assigning integrity values to RNA measurements. BMC Mol Biol 7: 3, 2006.

13. Dudoit S, Gentleman RC and Quackenbush J: Open source software for the analysis of microarray data. Biotechniques (Suppl): 45-51, 2003

14. Kooperberg C, Sipione S, LeBlanc M, et al: Evaluating test statistics to select interesting genes in microarray experiments. Hum Mol Genet 11: 2223-2232, 2002.

15. Golub TR, Slonim DK, Tamayo P, et al: Molecular classification of cancer: class discovery and class prediction by gene expression monitoring. Science 286: 531-537, 1999.

16. Birkenkamp-Demtroder K, Olesen SH, Sorensen FB, et al: Differential gene expression in colon cancer of the caecum versus the sigmoid and rectosigmoid. Gut 54: 374-384, 2005.

17. Barrier A, Lemoine A, Boelle PY, et al: Colon cancer prognosis prediction by gene expression profiling. Oncogene 24: 6155-6164, 2005.

18. Kruhoffer M, Jensen JL, Laiho P, et al: Gene expression signatures for colorectal cancer microsatellite status and HNPCC. Br J Cancer 92: 2240-2248, 2005.

19. Kleivi K, Lind GE, Diep CB, et al: Gene expression profiles of primary colorectal carcinomas, liver metastases, and carcinomatoses. Mol Cancer 6: 2, 2007.

20. Lin HM, Chatterjee A, Lin YH, et al: Genome wide expression profiling identifies genes associated with colorectal liver metastasis. Oncol Rep 17: 1541-1549, 2007. 
21. Wiese AH, Auer J, Lassmann S, et al: Identification of gene signatures for invasive colorectal tumor cells. Cancer Detect Prev 31: 282-295, 2007.

22. Wang Y, Jatkoe T, Zhang Y, et al: Gene expression profiles and molecular markers to predict recurrence of Dukes' B colon cancer. J Clin Oncol 22: 1564-1571, 2004.

23. Del Rio M, Molina F, Bascoul-Mollevi C, et al: Gene expression signature in advanced colorectal cancer patients select drugs and response for the use of leucovorin, fluorouracil, and irinotecan. J Clin Oncol 25: 773-780, 2007.

24. Cavalieri D, Dolara P, Mini E, et al: Analysis of gene expression profiles reveals novel correlations with the clinical course of colorectal cancer. Oncol Res 16: 535-548, 2007.

25. Kim IJ, Lim SB, Kang HC, et al: Microarray gene expression profiling for predicting complete response to preoperative chemoradiotherapy in patients with advanced rectal cancer. Dis Colon Rectum 50: 1342-1353, 2007.

26. Croner RS, Peters A, Brueckl WM, et al: Microarray versus conventional prediction of lymph node metastasis in colorectal carcinoma. Cancer 104: 395-404, 2005.

27. Croner RS, Fortsch T, Bruckl WM, et al: Molecular signature for lymphatic metastasis in colorectal carcinomas. Ann Surg 247: 803-810, 2008

28. Komori T, Takemasa I, Yamasaki M, et al: Gene expression of colorectal cancer: preoperative genetic diagnosis using endoscopic biopsies. Int J Oncol 32: 367-375, 2008.
29. Kwon HC, Kim SH, Roh MS, et al: Gene expression profiling in lymph node-positive and lymph node-negative colorectal cancer. Dis Colon Rectum 47: 141-152, 2004.

30. Chung S, Kim M, Choi W, et al: Expression of translationally controlled tumor protein mRNA in human colon cancer. Cancer Lett 156: 185-190, 2000.

31. Stojadinovic A, Hooke JA, Shriver CD, et al: HYOU1/Orp150 expression in breast cancer. Med Sci Monit 13: BR231-BR239, 2007.

32. Didelot C, Schmitt E, Brunet M, et al: Heat shock proteins: endogenous modulators of apoptotic cell death. Handb Exp Pharmacol 172: 171-198, 2006

33. Andreasson C, Fiaux J, Rampelt H, et al: Hsp110 is a nucleotide-activated exchange factor for Hsp70. J Biol Chem 283: 8877-8884, 2008

34. Facciponte JG, Wang XY and Subjeck JR: Hsp110 and Grp170, members of the Hsp70 superfamily, bind to scavenger receptorA and scavenger receptor expressed by endothelial cells-I. Eur J Immunol 37: 2268-2279, 2007.

35. Hosaka S, Nakatsura T, Tsukamoto H, et al: Synthetic small interfering RNA targeting heat shock protein 105 induces apoptosis of various cancer cells both in vitro and in vivo. Cancer Sci 97: 623-632, 2006.

36. Kai M, Nakatsura T, Egami H, et al: Heat shock protein 105 is overexpressed in a variety of human tumors. Oncol Rep 10 : 1777-1782, 2003. 